\title{
Plasma Endocan as a Predictor of Cardiovascular Event in Patients with End-Stage Renal Disease on Hemodialysis
}

\author{
Jin Sug Kim ${ }^{1,+}$, Gang Jee Ko ${ }^{2,+}$, Yang Gyun Kim ${ }^{1}{ }^{\mathbb{D}}$, So Young Lee ${ }^{3}$, Dong Young Lee ${ }^{4}$, \\ Kyung Hwan Jeong ${ }^{1, *}$ and Sang Ho Lee ${ }^{1, *}$ \\ 1 Division of Nephrology, Department of Internal Medicine, Kyung Hee University School of Medicine, \\ Seoul 02453, Korea; jinsuk0902@naver.com (J.S.K.); apple8840@hanmail.net (Y.G.K.) \\ 2 Division of Nephrology, Department of Internal Medicine, Korea University College of Medicine, \\ Seoul 02841, Korea; lovesba@hanmail.net \\ 3 Division of Nephrology, Department of Internal Medicine, CHA Bundang Medical Center, CHA University, \\ Seongnam 13496, Korea; ysy0119@cha.ac.kr \\ 4 Division of Nephrology, Department of Internal Medicine, Veterans Health Service Medical Center, \\ Seoul 05368, Korea; biizz@hanmail.net \\ * Correspondence: khjeong@khu.ac.kr (K.H.J.); lshkidney@khu.ac.kr (S.H.L.); Tel.: +82-2-958-8200 (K.H.J.); \\ +82-440-6121 (S.H.L.) \\ + Jin Sug Kim and Gang Jee Ko contributed to this work equally.
}

Received: 17 November 2020; Accepted: 15 December 2020; Published: 18 December 2020

\begin{abstract}
Endocan, a potential biomarker of endothelial dysfunction, is associated with increased cardiovascular risk. We investigated the utility of plasma endocan for predicting cardiovascular risk in end-stage renal disease (ESRD) patients undergoing hemodialysis. Of the 452 patients in the K-cohort, 354 with available plasma endocan levels were enrolled. The correlation between plasma endocan levels and the clinical characteristics of a study population were analyzed. We divided patients into two groups, according to plasma endocan levels, and investigated the predictive value of endocan for the occurrence of cardiovascular events. In a multiple linear regression analysis, plasma endocan levels were positively correlated with previous cardiovascular events and negatively correlated with body mass index, albumin, and triglyceride. Patients with higher plasma endocan levels experienced more frequent cardiovascular events than those with lower plasma endocan levels (12.9\% in the lower group vs. $22.7 \%$ in the higher group, $p=0.016)$. Cox proportional hazard models showed that higher plasma endocan levels were an independent predictor of cardiovascular events in ESRD patients on hemodialysis ((hazard ration) HR 1.949, 95\% (confidence interval) CI 1.144-3.319, $p=0.014$ ). Our results suggest that plasma endocan level might be a useful biomarker for predicting cardiovascular events in ESRD patients on hemodialysis.
\end{abstract}

Keywords: endocan; hemodialysis; end-stage renal disease; biomarker

\section{Introduction}

Patients with end-stage renal disease (ESRD) on hemodialysis have an increased mortality rate compared to the general population. Cardiovascular disease is the most common cause of death, accounting for about $40 \%$ to $50 \%$ of mortalities in ESRD patients [1]. In addition to traditional cardiovascular risk factors, such as hypertension, diabetes, and dyslipidemia, additional risk factors, including volume overload and disturbance of calcium and phosphate metabolism, can contribute to increased cardiovascular risk in ESRD patients [1,2]. A predictive tool for assessing cardiovascular risk 
in patients with ESRD would be beneficial. However, limited data are available on biological markers capable of predicting cardiovascular outcomes in patients with ESRD.

Endocan, a circulating proteoglycan secreted from vascular endothelial cells, affects the regulation of cell adhesion, migration, proliferation, and neovascularization in the endothelium [3,4]. Several studies have demonstrated that endocan is involved in endothelial dysfunction and inflammation, and could be an independent risk factor for poor clinical outcomes in various disease conditions [5-8]. Our research group previously reported that endocan is a potential biomarker of disease progression in patients with IgA nephropathy [9] and microvascular inflammation in kidney transplant patients [10]. Recently, endocan has emerged as a promising biomarker of cardiovascular disease. One meta-analysis demonstrated that serum endocan levels were significantly increased in patients with cardiovascular disease and suggested that endocan is a risk factor for cardiovascular disease [11]. Previous studies reported that higher endocan levels were associated with adverse cardiovascular outcomes in patients with various disease conditions, such as acute myocardial infarction, hypertension, and chronic kidney disease (CKD) [12-14]. However, to our knowledge, no study has been conducted to investigate the role of endocan in predicting cardiovascular risk in ESRD patients on hemodialysis.

In this study, we measured the levels of plasma endocan and analyzed its association with clinical characteristics in ESRD patients on hemodialysis. We also investigated the predictive value of endocan for cardiovascular outcomes based on more than two years of follow-up observations.

\section{Materials and Methods}

\subsection{Study Population and Design}

The study population was derived from the K-cohort, a multicenter, Internet-based, prospective cohort of patients with ESRD on hemodialysis in South Korea. The K-cohort was developed to evaluate the mortality and morbidity of ESRD patients, starting from 2016 (CRIS no. KCT0003281). ESRD patients from six hospitals, who were over 18 years of age and on hemodialysis, were included in the K-cohort if they were undergoing hemodialysis three times a week for over three months. Inclusion/exclusion criteria and detailed cohort design have been described previously $[15,16]$. Of the 452 patients who were enrolled as part of the K-cohort from June 2016 to August 2019, 354 with available plasma endocan data were included in this study. The study population was divided into two groups: lower endocan and higher endocan groups. We compared baseline characteristics and clinical outcomes between the two groups, and analyzed the association of plasma endocan levels with clinical parameters. We also investigated the predictive value of endocan for adverse cardiovascular outcomes.

The study was conducted in accordance with the Declaration of Helsinki, and the institutional review board of each participating center approved the study protocol (KHNMC IRB No. 2016-04-039). Written consent was obtained from all participants involved in this study before enrollment.

\subsection{Variables and Study Outcome}

We comprehensively collected baseline variables, including age, sex, body mass index (BMI), prior medical history, comorbid conditions, dialysis information, laboratory data, and medications. Blood samples for laboratory data were collected in the fasting state, just before the start of hemodialysis in a midweek session. The primary study outcome was a cardiovascular event, which was defined as the development of at least one of the following conditions: acute coronary syndrome (ACS), stable angina requiring percutaneous coronary intervention (PCI) or coronary artery bypass grafting (CABG), heart failure, ventricular arrhythmia, cardiac arrest, and sudden death. Disease-free survival was measured from the date of enrollment to the first occurrence of composite cardiovascular outcomes. All patients were monitored for the development of study outcomes until March 2020. 


\subsection{Laboratory Analysis}

Routine biochemical laboratory parameters were measured using standard methods. We collected plasma samples using tubes treated with ethylenediaminetetraacetic acid (EDTA) on the day of enrolment in the study. After centrifugation for $15 \mathrm{~min}$ at $1000 \times \mathrm{g}$ at room temperature, samples were stored at $-80{ }^{\circ} \mathrm{C}$ until analysis. We measured endocan levels using an enzyme-linked immunosorbent assay (ELISA) using Magnetic Luminex ${ }^{\circledR}$ Screening Assay multiplex kits (R\&D Systems, Minneapolis, MN, USA), as described previously $[9,10]$.

\subsection{Statistical Analysis}

Continuous variables are described as means \pm standard deviations (SDs) and were analyzed using Student's t-test or the Mann-Whitney U test. Categorical variables are presented as frequencies and percentages. Categorical data were analyzed using the chi-square test or Fisher's exact test. Linear regression analyses were performed to assess the correlations between baseline clinical characteristics. The R package MaxStat (R Foundation for Statistical Computing, Vienna, Austria) was used to determine the optimal endocan cut-off value. In an analysis of all patients, a cut-off value of $1023.52 \mathrm{pg} / \mathrm{mL}$ was found to have the highest log-rank statistic of any cut-off value. Kaplan-Meier estimates of time to event endpoints were calculated. Multivariate Cox regression analyses were performed with all univariate associations of $p<0.1$ to identify a predictive model for cardiovascular event risk. The results are presented as hazard ratios (HRs) and $\pm 95 \%$ confidence intervals (CIs), and statistical significance is indicated. Statistical analyses were conducted using SPSS software version 19.0 (SPSS Inc., Chicago, IL, USA) and R software version R 4.0.2 (R Foundation for Statistical Computing, Vienna, Austria). A $p$-value $<0.05$ was considered to indicate statistical significance.

\section{Results}

\subsection{Baseline Clinical Characteristics}

A total of 354 adult patients with ESRD who were undergoing hemodialysis at six hospitals were enrolled in this study. Patients were divided into two groups, according to the optimal cut-off value of plasma endocan levels, which were obtained using the aforementioned method: a lower endocan group (endocan $1023.52 \mathrm{pg} / \mathrm{mL}, n=178$ ) and a higher endocan group (endocan $\geq 1023.52 \mathrm{pg} / \mathrm{mL}, n=176$ ). Baseline characteristics of the study population, according to plasma endocan levels, are summarized in Table 1. Patients in the higher endocan group had a lower BMI $(p<0.001)$ and a lower prevalence of diabetes mellitus $(p=0.001)$. Albumin and glucose levels were significantly lower in the higher endocan group than in the lower endocan group ( $p=0.032$ and $p=0.012$, respectively). Patients with higher endocan levels had longer hemodialysis duration $(p<0.001)$ and higher single-pool Kt/V $(p=0.013)$ than patients with lower endocan levels. There was a significant difference in lipid profiles and medications between the two groups.

Table 1. Baseline characteristics according to plasma endocan level.

\begin{tabular}{cccc}
\hline & $\begin{array}{c}\text { Lower Endocan Group } \\
(\boldsymbol{n = 1 7 8 )}\end{array}$ & $\begin{array}{c}\text { Higher Endocan Group } \\
(\boldsymbol{n}=\mathbf{1 7 6})\end{array}$ & $\boldsymbol{p}$ \\
\cline { 2 - 3 } & $\mathbf{< 1 0 2 3 . 5 2} \mathbf{~ p g} / \mathbf{m L}$ & $\mathbf{1 0 2 3 . 5 2} \mathbf{~ p g} / \mathbf{m L}$ & \\
\hline Age (year) & $60.84 \pm 13.53$ & $63.45 \pm 11.82$ & 0.054 \\
Sex (Male, $\%)$ & $126(70.8 \%)$ & $109(61.9 \%)$ & 0.078 \\
Body mass index $\left(\mathrm{kg} / \mathrm{m}^{2}\right)$ & $24.35 \pm 4.20$ & $21.78 \pm 3.18$ & $<0.001$ \\
Hypertension $(n, \%)$ & $154(86.5 \%)$ & $149(84.7 \%)$ & 0.619 \\
Diabetes mellitus $(n, \%)$ & $115(64.6 \%)$ & $83(47.2 \%)$ & 0.001 \\
Previous cardiovascular event $(n, \%)$ & $67(37.6 \%)$ & $82(46.6 \%)$ & 0.088 \\
\hline
\end{tabular}


Table 1. Cont.

\begin{tabular}{|c|c|c|c|}
\hline & $\begin{array}{l}\text { Lower Endocan Group } \\
\qquad(n=178)\end{array}$ & $\begin{array}{l}\text { Higher Endocan Group } \\
\qquad(n=176)\end{array}$ & \multirow[t]{2}{*}{$p$} \\
\hline & $<1023.52 \mathrm{pg} / \mathrm{mL}$ & $\geq 1023.52 \mathrm{pg} / \mathrm{mL}$ & \\
\hline Cause of ESRD $(n, \%)$ & & & 0.024 \\
\hline Diabetes mellitus & $97(54.5 \%)$ & $69(39.2 \%)$ & \\
\hline Hypertension & $25(14.0 \%)$ & $47(26.7 \%)$ & \\
\hline Glomerulonephritis & $20(11.2 \%)$ & $22(12.5 \%)$ & \\
\hline ADPKD & $11(6.2 \%)$ & $8(4.5 \%)$ & \\
\hline Others & $21(11.8 \%)$ & $27(15.3 \%)$ & \\
\hline Unknown & $4(2.2 \%)$ & $3(1.7 \%)$ & \\
\hline HD duration (month) & $46.62 \pm 53.01$ & $72.77 \pm 76.65$ & $<0.001$ \\
\hline Average ultrafiltration (L) & $2.31 \pm 1.06$ & $2.17 \pm 1.05$ & 0.222 \\
\hline Single-pool Kt/V & $1.53 \pm 0.29$ & $1.61 \pm 0.30$ & 0.013 \\
\hline Pre-dialysis SBP (mmHg) & $141.69 \pm 19.58$ & $145.53 \pm 20.55$ & 0.073 \\
\hline Pre-dialysis DBP (mmHg) & $72.14 \pm 12.43$ & $73.09 \pm 13.55$ & 0.492 \\
\hline \multicolumn{4}{|l|}{ Laboratory findings } \\
\hline Hemoglobin (g/dL) & $10.52 \pm 1.24$ & $10.34 \pm 1.21$ & 0.187 \\
\hline Albumin $(\mathrm{g} / \mathrm{dL})$ & $3.85 \pm 0.31$ & $3.78 \pm 0.34$ & 0.032 \\
\hline Creatinine (mg/dL) & $9.46 \pm 2.87$ & $9.31 \pm 2.79$ & 0.615 \\
\hline $\mathrm{Ca} \times \mathrm{P}$ & $41.37 \pm 12.05$ & $40.20 \pm 12.98$ & 0.379 \\
\hline Glucose (mg/dL) & $161.91 \pm 66.20$ & $145.19 \pm 57.41$ & 0.012 \\
\hline Total cholesterol (mg/dL) & $141.01 \pm 31.62$ & $130.48 \pm 27.42$ & 0.001 \\
\hline Triglyceride (mg/dL) & $147.46 \pm 90.70$ & $93.30 \pm 46.42$ & $<0.001$ \\
\hline LDL cholesterol (mg/dL) & $80.48 \pm 26.70$ & $73.04 \pm 24.45$ & 0.007 \\
\hline HDL cholesterol (mg/dL) & $42.46 \pm 13.17$ & $47.13 \pm 12.27$ & 0.001 \\
\hline hs-CRP (mg/dL) & $3.91 \pm 8.29$ & $3.88 \pm 7.89$ & 0.973 \\
\hline \multicolumn{4}{|l|}{ Medications $(n, \%)$} \\
\hline ARB or ACEi & $102(57.3 \%)$ & $108(61.4 \%)$ & 0.437 \\
\hline $\mathrm{CCB}$ & $104(58.45)$ & $113(64.2 \%)$ & 0.264 \\
\hline Beta-blocker & $60(33.7 \%)$ & $90(51.1 \% 0$ & 0.001 \\
\hline Statin & $83(46.6 \%)$ & $83(47.2 \%)$ & 0.920 \\
\hline Anti-platelet drugs & $124(69.7 \%)$ & $128(72.7 \%)$ & 0.524 \\
\hline Anti-diabetic drugs & $95(54.3 \%)$ & $60(34.3 \%)$ & $<0.001$ \\
\hline
\end{tabular}

ESRD, end-stage renal disease; ADPKD, autosomal dominant polycystic kidney disease; HD, hemodialysis; SBP, systolic blood pressure; DBP, diastolic blood pressure; LDL, low-density lipoprotein; HDL, high-density lipoprotein; hs-CRP, high-sensitivity C-reactive protein; ARB, angiotensin II receptor blocker; ACEi, angiotensin-converting enzyme inhibitor; $\mathrm{CCB}$, calcium channel blocker.

\subsection{Associations between Plasma Endocan Level and Baseline Characteristics}

Table 2 shows associations between plasma endocan level and various clinical characteristics in patients with ESRD on hemodialysis. In univariate linear regression analyses, hemodialysis duration, single-pool Kt/V, and HDL-cholesterol were positively associated with plasma endocan level. Plasma endocan level was inversely associated with BMI, diabetes mellitus, albumin, glucose, total cholesterol, and triglyceride. Variables with $p$-values $<0.10$ in the univariate analysis were included for multivariate analysis. In multivariate linear regression analysis, endocan level was positively associated with a previous cardiovascular event $(\beta=0.106 ; p=0.039)$ and negatively associated with $\operatorname{BMI}(\beta=-0.170 ; p=0.005)$, albumin $(\beta=-0.118 ; p=0.026)$, and triglyceride $(\beta=-0.155 ; p=0.025)$. 
Table 2. Association between plasma endocan level and various clinical characteristics.

\begin{tabular}{|c|c|c|c|c|c|c|c|c|}
\hline & \multicolumn{4}{|c|}{ Simple Regression } & \multicolumn{4}{|c|}{ Multiple Regression } \\
\hline & B & SE & Beta & $p$ & B & SE & Beta & $p$ \\
\hline Age (years) & 3.262 & 2.602 & 0.067 & 0.211 & & & & \\
\hline Male sex & -68.797 & 70.252 & -0.052 & 0.328 & & & & \\
\hline Body mass index $\left(\mathrm{kg} / \mathrm{m}^{2}\right)$ & -44.83 & 8.097 & -0.283 & $<0.001$ & -26.871 & 9.535 & -0.170 & 0.005 \\
\hline Hypertension & 47.018 & 94.602 & 0.026 & 0.619 & & & & \\
\hline Diabetes mellitus & -217.213 & 55.928 & -0.173 & 0.001 & -45.131 & 108.377 & -0.036 & 0.680 \\
\hline Previous cardiovascular event & 128.925 & 66.96 & 0.102 & 0.055 & 134.133 & 64.726 & 0.106 & 0.039 \\
\hline HD duration (month) & 1.591 & 0.491 & 0.171 & 0.001 & 0.644 & 0.511 & 0.069 & 0.209 \\
\hline Average ultrafiltration (L) & -17.422 & 31.454 & -0.03 & 0.58 & & & & \\
\hline Single-pool Kt/V & 243.421 & 111.197 & 0.116 & 0.029 & -76.845 & 124.517 & -0.036 & 0.538 \\
\hline Pre-dialysis SBP (mmHg) & 3.059 & 1.645 & 0.099 & 0.064 & 2.962 & 1.755 & 0.095 & 0.092 \\
\hline Pre-dialysis DBP (mmHg) & 4.726 & 2.548 & 0.098 & 0.065 & 3.224 & 2.768 & 0.067 & 0.245 \\
\hline \multicolumn{9}{|l|}{ Laboratory findings } \\
\hline Hemoglobin (g/dL) & -54.434 & 26.931 & -0.107 & 0.044 & -22.458 & 27.032 & -0.044 & 0.407 \\
\hline Albumin (g/dL) & -264.965 & 100.896 & -0.139 & 0.009 & -225.804 & 101.063 & -0.118 & 0.026 \\
\hline Creatinine $(\mathrm{mg} / \mathrm{dL})$ & -6.573 & 11.764 & -0.03 & 0.577 & & & & \\
\hline $\mathrm{Ca} \times \mathrm{P}$ & -3.18 & 2.653 & -0.064 & 0.231 & & & & \\
\hline Glucose (mg/dL) & -1.593 & 0.526 & -0.159 & 0.003 & -0.907 & 0.606 & -0.091 & 0.136 \\
\hline Total cholesterol (mg/dL) & -2.308 & 1.101 & -0.111 & 0.037 & -0.497 & 2.178 & -0.024 & 0.820 \\
\hline Triglyceride (mg/dL) & -2.554 & 0.41 & -0.315 & $<0.001$ & -1.253 & 0.555 & -0.155 & 0.025 \\
\hline LDL cholesterol (mg/dL) & -2.244 & 1.282 & -0.093 & 0.081 & 0.214 & 2.233 & 0.009 & 0.923 \\
\hline HDL cholesterol (mg/dL) & 8.838 & 2.531 & 0.183 & 0.001 & 3.447 & 3.492 & 0.070 & 0.324 \\
\hline hs-CRP (mg/dL) & 1.676 & 4.115 & 0.022 & 0.684 & & & & \\
\hline \multicolumn{9}{|l|}{ Medications } \\
\hline $\mathrm{ARB}$ or $\mathrm{ACEi}$ & 98.081 & 67.448 & 0.077 & 0.147 & & & & \\
\hline CCB & 111.872 & 69.029 & 0.086 & 0.106 & & & & \\
\hline Beta-blocker & 91.817 & 67.072 & 0.073 & 0.172 & & & & \\
\hline Statin & -21.543 & 66.583 & -0.017 & 0.746 & & & & \\
\hline Anti-platelet drugs & -23.996 & 73.365 & -0.327 & 0.744 & & & & \\
\hline Anti-diabetic drugs & -201.941 & 66.601 & -0.160 & 0.003 & -30.054 & 104.717 & -0.024 & 0.774 \\
\hline
\end{tabular}

HD, hemodialysis; SBP, systolic blood pressure; DBP, diastolic blood pressure; LDL, low-density lipoprotein; HDL, high-density lipoprotein; hs-CRP, high-sensitivity C-reactive protein; ARB, angiotensin II receptor blocker; ACEi, angiotensin-converting enzyme inhibitor; $\mathrm{CCB}$, calcium channel blocker.

\subsection{Study Outcome in Accordance with Plasma Endocan Level}

The prevalence of cardiovascular events, according to plasma endocan levels, is shown in Table 3. The mean follow-up duration was 34.56 months. During the follow-up period, 63 (17.8\%) patients experienced cardiovascular events. Of the 63 patients, 19 (5.4\%) had ACS, 11 (3.1\%) had stable angina, requiring PCI or CABG, $7(2.0 \%)$ had heart failure, $3(0.8 \%)$ had ventricular arrhythmia, and $23(6.5 \%)$ had cardiac death. Patients with higher plasma endocan levels frequently experienced cardiovascular events $(12.9 \%$ in the lower group vs. $22.7 \%$ in the higher group, $p=0.016)$. Figure 1 shows cardiovascular event-free survival, according to plasma endocan levels. The log-rank test identified a significant association between the endocan level and cardiovascular event-free survival ( $p=0.006)$. To analyze the cause of death, we also observed the prevalence of non-cardiac death. Thirty-seven $(10.5 \%)$ patients experienced non-cardiac death and the most common cause of non-cardiac death was infection. Patients with higher plasma endocan levels showed a more frequent prevalence of non-cardiac death (6.2\% in the lower group vs. $14.8 \%$ in the higher group, $p=0.008)$ (Table S1).

Univariate and multivariate Cox regression analyses were conducted to identify risk factors associated with cardiovascular events in ESRD patients on hemodialysis (Table 4). In univariate Cox regression analysis, diabetes mellitus (HR 2.287, 95\% CI 1.1311-3.990, $p=0.004)$, previous cardiovascular events (HR 2.308, 95\% CI, 1.392-3.825, $p=0.001$ ), glucose (HR 1.005, 95\% CI, 1.002-1.008, $p=0.004$ ), use of angiotensin II receptor blockers or angiotensin-converting enzyme inhibitor (HR 1.746, 95\% CI 1.011-3.016, $p=0.046$ ), use of calcium channel blocker (HR 1.864, 95\% CI 1.044-3.329, $p=0.035$ ), use of statin (HR 1.701, 95\% CI 1.030-2.811, $p=0.038$ ), use of antidiabetic drugs (HR 2.018, 95\% CI 1.218-3.345, $p=0.006$ ), and higher plasma endocan levels (HR 1.994, 95\% CI, 1.193-3.334, $p=0.008$ ) showed a significant association with the development of cardiovascular events in ESRD patients on hemodialysis. 
After adjustment for variables with a $p$-value $<0.10$ in the univariate analysis, previous cardiovascular events (HR 1.976, 95\% CI, 1.139-3.430, $p=0.015$ ) and higher plasma endocan levels (HR 1.949, 95\% CI 1.144-3.319, $p=0.014$ ) were independent factors associated with the development of cardiovascular events in patients with ESRD on hemodialysis.

Table 3. Clinical outcomes according to plasma endocan level.

\begin{tabular}{ccccc}
\hline & Overall & $\begin{array}{c}\text { Lower Endocan } \\
\text { Group }\end{array}$ & $\begin{array}{c}\text { Higher Endocan } \\
\text { Group }\end{array}$ & $\boldsymbol{p}$ \\
\cline { 2 - 4 } & $(\boldsymbol{n = 3 5 4 )}$ & $(\boldsymbol{n = 1 7 8 )}$ & $\mathbf{n = 1 7 6 )}$ & \\
\hline Follow-up duration (month) & $34.56 \pm 13.86$ & $35.99 \pm 13.69$ & $33.10 \pm 13.92$ & 0.051 \\
Cardiovascular events & $63(17.8 \%)$ & $23(12.9 \%)$ & $40(22.7 \%)$ & 0.016 \\
Acute coronary syndrome & $19(5.4 \%)$ & $7(3.9 \%)$ & $12(6.8 \%)$ & \\
Stable angina with PCI or CABG & $11(3.1 \%)$ & $4(2.2 \%)$ & $7(4.0 \%)$ \\
Heart failure & $7(2.0 \%)$ & $2(1.1 \%)$ & $5(2.8 \%)$ \\
Ventricular arrhythmia & $3(0.8 \%)$ & $1(0.6 \%)$ & $2(1.1 \%)$ \\
Cardiac death or sudden death & $23(6.5 \%)$ & $9(5.1 \%)$ & $14(8.0 \%)$ \\
\hline
\end{tabular}

$\mathrm{PCI}$, percutaneous coronary intervention; $\mathrm{CABG}$, coronary artery bypass graft.

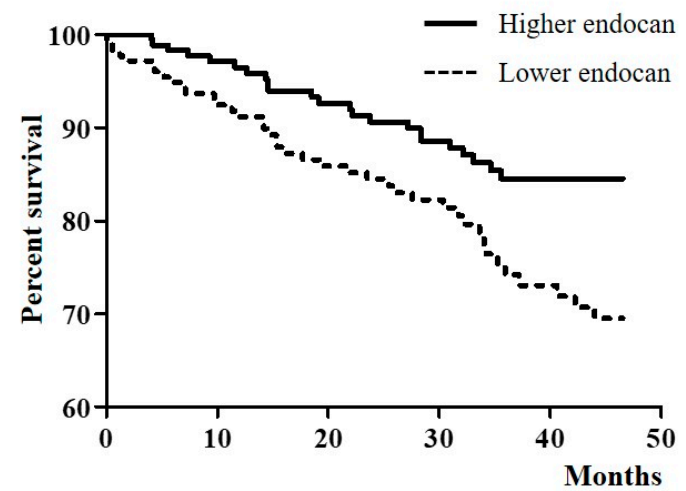

Figure 1. Cardiovascular event-free survival according to plasma endocan level.

Table 4. Predictors of cardiovascular events in univariate and multivariate Cox regression analyses.

\begin{tabular}{|c|c|c|c|c|}
\hline \multirow{2}{*}{ Variable } & Univariate Analysis & \multirow{2}{*}{$p$} & Multivariate Analysis & \multirow{2}{*}{$p$} \\
\hline & HR $(95 \%$ CI $)$ & & HR $(95 \%$ CI $)$ & \\
\hline Age (year) & $1.017(0.998-1.036)$ & 0.075 & $1.007(0.986-1.030)$ & 0.512 \\
\hline Male sex & $0.774(0.465-1.286)$ & 0.322 & & \\
\hline Body mass index $\left(\mathrm{kg} / \mathrm{m}^{2}\right)$ & $0.976(0.916-1.040)$ & 0.453 & & \\
\hline Hypertension & $2.090(0.838-5.211)$ & 0.114 & & \\
\hline Diabetes mellitus & $2.287(1.311-3.990)$ & 0.004 & $1.554(0.656-3.685)$ & 0.316 \\
\hline Previous cardiovascular event & $2.308(1.392-3.825)$ & 0.001 & $1.976(1.139-3.430)$ & 0.015 \\
\hline HD duration (month) & $0.998(0.994-1.002)$ & 0.409 & & \\
\hline Average ultrafiltration (L) & $0.987(0.781-1.247)$ & 0.987 & & \\
\hline Single-pool Kt/V & $0.639(0.278-1.468)$ & 0.291 & & \\
\hline Pre-dialysis SBP (mmHg) & $1.005(0.993-1.018)$ & 0.398 & & \\
\hline Pre-dialysis DBP (mmHg) & $0.991(0.971-1.012)$ & 0.395 & & \\
\hline \multicolumn{5}{|l|}{ Laboratory findings } \\
\hline Hemoglobin (g/dL) & $0.870(0.719-1.052)$ & 0.15 & & \\
\hline Albumin (g/dL) & $0.621(0.288-1.343)$ & 0.226 & & \\
\hline Creatinine $(\mathrm{mg} / \mathrm{dL})$ & $0.929(0.848-1.019)$ & 0.118 & & \\
\hline $\mathrm{Ca} \times \mathrm{P}$ & $0.996(0.975-1.017)$ & 0.716 & & \\
\hline
\end{tabular}


Table 4. Cont.

\begin{tabular}{|c|c|c|c|c|}
\hline \multirow{2}{*}{ Variable } & Univariate Analysis & \multirow{2}{*}{$p$} & Multivariate Analysis & \multirow{2}{*}{$p$} \\
\hline & $\operatorname{HR}(95 \% \mathrm{CI})$ & & HR (95\% CI) & \\
\hline Glucose $(\mathrm{mg} / \mathrm{dL})$ & $1.005(1.002-1.008)$ & 0.004 & $1.002(0.998-1.006)$ & 0.286 \\
\hline Total cholesterol (mg/dL) & $0.994(0.985-1.003)$ & 0.164 & & \\
\hline Triglyceride (mg/dL) & $1.001(0.997-1.004)$ & 0.736 & & \\
\hline LDL cholesterol (mg/dL) & $0.998(0.988-1.008)$ & 0.962 & & \\
\hline HDL cholesterol (mg/dL) & $0.992(0.973-1.012)$ & 0.425 & & \\
\hline hs-CRP (mg/dL) & $0.997(0.965-1.031)$ & 0.882 & & \\
\hline \multicolumn{5}{|l|}{ Medications } \\
\hline $\mathrm{ARB}$ or $\mathrm{ACEi}$ & $1.746(1.011-3.016)$ & 0.046 & $1.292(0.712-2.345)$ & 0.399 \\
\hline $\mathrm{CCB}$ & $1.864(1.044-3.329)$ & 0.035 & $1.724(0.911-3.262)$ & 0.095 \\
\hline Beta-blocker & $1.038(0.630-1.709)$ & 0.885 & & \\
\hline Statin & $1.701(1.030-2.811)$ & 0.038 & $1.295(0.766-2.191)$ & 0.334 \\
\hline Antiplatelet drugs & $1.557(0.846-2.866)$ & 0.155 & & \\
\hline Antidiabetic drugs & $2.018(1.218-3.345)$ & 0.006 & $1.296(0.588-2.858)$ & 0.520 \\
\hline \multicolumn{5}{|l|}{ Endocan levels } \\
\hline Lower & 1 & & 1 & \\
\hline Higher & $2.027(1.213-3.389)$ & 0.007 & 1.949 (1.144-3.319) & 0.014 \\
\hline
\end{tabular}

HD, hemodialysis; SBP, systolic blood pressure; DBP, diastolic blood pressure; LDL, low-density lipoprotein; HDL, high-density lipoprotein; hs-CRP, high-sensitivity C-reactive protein; ARB, angiotensin II receptor blocker; ACEi, angiotensin-converting enzyme inhibitor; $\mathrm{CCB}$, calcium channel blocker.

\section{Discussion}

In this study, we measured plasma endocan levels and investigated its correlation with clinical characteristics in ESRD patients on hemodialysis, using a prospective multicenter cohort. We also determined the predictive value of plasma endocan levels on the development of cardiovascular events in ESRD patients on hemodialysis. Our principal findings are that, in patients with ESRD undergoing hemodialysis, (1) plasma endocan levels were positively correlated with previous cardiovascular events and negatively correlated with BMI, albumin, and triglyceride; (2) patients with higher plasma endocan levels experienced cardiovascular events more frequently than those with lower plasma endocan levels; (3) higher plasma endocan level was an independent predictor of cardiovascular events, even after adjusting for various clinical parameters.

Endocan has been reported to be associated with the occurrence and clinical outcomes of various disease conditions, such as hypertension, sepsis, and malignancy [17-20]. The association between endocan and kidney diseases has also been studied. Gunay et al. [21] reported that serum endocan levels were significantly increased in patients with acute kidney injury (AKI). Other researchers have suggested serum endocan as a potential biomarker for differentiating causes of intrinsic AKI [22]. Yilmaz et al. [6] demonstrated that plasma endocan levels are correlated with decreasing estimated glomerular filtration rate, and influence cardiovascular events, and all-cause mortality in non-dialysis patients with CKD.

The clinical relevance of endocan has also been analyzed in ESRD patients who needed renal replacement therapy. In kidney transplant patients, serum endocan levels were significantly correlated with chronic renal allograft injury [23]. In our previous study, plasma and urinary endocan levels were significantly increased in kidney transplant patients with acute antibody-mediated rejection, and patients with high endocan levels showed worse renal survival [10]. In ESRD patients undergoing peritoneal dialysis, serum endocan levels have a predictive value for decreasing urine volume and showed a significant correlation with clinical characteristics and cardiovascular events [24,25]. However, only a few studies have evaluated endocan in hemodialysis patients [26,27], and the studies were conducted with a relatively small sample size, and did not analyze the association between clinical outcomes and endocan levels. 
In the present study, we demonstrated the clinical relevance of endocan in patients with ESRD on hemodialysis. We found that endocan was inversely correlated with BMI, albumin, and triglyceride levels. Given that these factors are widely accepted as showing nutritional status in hemodialysis patients, endocan might be a potential indicator of nutritional status in patients with ESRD on hemodialysis. To determine the association between endocan level and nutritional status, we calculated the geriatric nutritional risk index (GNRI) [28] and performed further analyses. GNRI was significantly lower in the higher endocan group than in the lower endocan group $(91.89 \pm 7.42$ in the lower endocan group and $87.17 \pm 7.13$ in the higher endocan group, $p<0.001)$. Endocan levels showed a negative correlation with GNRI $(r=-0.294, p<0.001)$. Similar to our findings, Poon et al. [25] reported that serum endocan levels correlated with the nutritional status of ESRD patients on peritoneal dialysis. In their study, serum endocan levels were inversely correlated with serum albumin levels, and patients with higher endocan levels had a lower subjective global assessment scale and a higher malnutrition-inflammation score. Further studies are needed to establish the mechanism that underlies the relationship between nutritional status and endocan levels.

In our study, the lower endocan group showed significantly lower single-pool Kt/V compared with the higher endocan group (1.53 \pm 0.29 in the lower endocan group and $1.61 \pm 0.30$ in the higher endocan group, $p=0.013$ ). Although no further analysis was conducted on this result, the following supposition can be made: given that patients in the lower endocan group have shorter hemodialysis (HD) duration than those in the higher endocan group, it is assumed that patients in the lower endocan group have a higher residual renal function. Previous studies reported that endocan levels showed a negative association with residual renal function [24,25]. The result of adding residual renal function to assess dialysis adequacy may differ from our prior result. However, we could not measure residual renal function due to the limitation of cohort design. To overcome this limitation, we are planning an additional cohort study with the modification of cohort design.

The results of the present study suggest that plasma endocan levels can be used as an independent cardiovascular risk factor, consistent with previous studies. A recent meta-analysis showed that endocan levels were significantly increased in patients with cardiovascular disease [11]. In another study, plasma endocan levels were associated with cardiovascular events and all-cause mortality [29]. Pawlak et al. [14] showed that plasma endocan levels were significantly increased in patients with CKD, and independently associated with soluble intercellular adhesion molecule- 1 and soluble vascular cell adhesion molecule- 1 , which affect the prevalence of cardiovascular disease in these patients. Our findings provide important evidence of the utility of endocan as a predictive biomarker of cardiovascular risk in patients with ESRD on hemodialysis.

Our study had some potential limitations. Firstly, the plasma endocan level was measured only once, which may have resulted in the incorrect classification of patients. To overcome this limitation, we intend to establish an additional independent cohort to validate our findings. Secondly, the cut-off value of higher and lower serum endocan was chosen following the appropriate calculation; however, further studies with large sample sizes are needed to ascertain the reliability of the cut-off value. Thirdly, since the patients enrolled in this study were predominantly Korean, the results should be generalized with caution, and further studies are needed to assess the clinical relevance of endocan levels in different populations. However, our study is significant because it is the first study to demonstrate a significant association between plasma endocan levels and cardiovascular events in ESRD patients on hemodialysis, using a relatively large number of patients from a prospective multicenter cohort.

In conclusion, ESRD patients on hemodialysis with higher plasma endocan levels showed more frequent cardiovascular events than those with lower plasma endocan levels. Higher plasma endocan level was an independent predictor of cardiovascular events in patients with ESRD on hemodialysis. Plasma endocan levels could be a useful predictive marker of the risk of cardiovascular events in patients with ESRD on hemodialysis. Further studies involving multiethnic and/or larger numbers of participants are needed to ascertain the underlying mechanism and validate our findings. 
Supplementary Materials: The following are available online at http://www.mdpi.com/2077-0383/9/12/4086/s1, Table S1: Non-cardiac death according to plasma endocan level.

Author Contributions: Conceptualization, J.S.K., K.H.J., and S.H.L.; methodology, J.S.K., Y.G.K., and D.Y.L.; software, K.H.J. and S.H.L.; validation, S.Y.L. and G.J.K.; formal analysis, J.S.K., K.H.J., and S.H.L.; investigation, J.S.K., Y.G.K., S.Y.L., G.J.K., and D.Y.L.; resources, S.Y.L., G.J.K., D.Y.L., K.H.J., and S.H.L.; data curation, Y.G.K., and D.Y.L.; writing—original draft preparation, J.S.K.; writing-review and editing, G.J.K., K.H.J., and S.H.L.; visualization, J.S.K.; supervision, K.H.J., and S.H.L. All authors have read and agreed to the published version of the manuscript.

Funding: This research was supported by a grant from the Patient-Centered Clinical Research Coordinating Center, funded by the Ministry of Health \& Welfare, Republic of Korea (grant number: HI19C0481, HC19C0041). The funding organizations had no role in the design of the study, analysis, and interpretation of the data, nor in the writing of the manuscript.

Acknowledgments: The authors appreciate the support and cooperation of the staff of the participating centers.

Conflicts of Interest: The authors declare no conflict of interest.

\section{References}

1. Foley, R.N.; Parfrey, P.S.; Sarnak, M.J. Clinical epidemiology of cardiovascular disease in chronic renal disease. Am. J. Kidney Dis. 1998, 32, S112-S119. [CrossRef] [PubMed]

2. Santoro, A.; Mancini, E. Cardiac effects of chronic inflammation in dialysis patients. Nephrol. Dial. Transplant. 2002, 17, 10-15. [CrossRef] [PubMed]

3. Kali, A.; Shetty, K.R. Endocan: A novel circulating proteoglycan. Indian J. Pharmacol. 2014, 46, 579-583. [CrossRef] [PubMed]

4. Sarrazin, S.; Adam, E.; Lyon, M.; Depontieu, F.; Motte, V.; Landolfi, C.; Lortat-Jacob, H.; Bechard, D.; Lassalle, P.; Delehedde, M. Endocan or endothelial cell specific molecule-1 (ESM-1): A potential novel endothelial cell marker and a new target for cancer therapy. Biochim. Biophys. Acta Bioenerg. 2006, 1765, 25-37. [CrossRef] [PubMed]

5. Bessa, J.; Albino-Teixeira, A.; Reina-Couto, M.; Sousa, T. Endocan: A novel biomarker for risk stratification, prognosis and therapeutic monitoring in human cardiovascular and renal diseases. Clin. Chim. Acta 2020, 509, 310-335. [CrossRef] [PubMed]

6. Yilmaz, M.I.; Siriopol, D.; Saglam, M.; Kurt, Y.G.; Unal, H.U.; Eyileten, T.; Gok, M.; Cetinkaya, H.; Oguz, Y.; Sari, S.; et al. Plasma endocan levels associate with inflammation, vascular abnormalities, cardiovascular events, and survival in chronic kidney disease. Kidney Int. 2014, 86, 1213-1220. [CrossRef]

7. Pauly, D.; Hamed, S.; Behnes, M.; Lepiorz, D.; Lang, S.; Akin, I.; Borggrefe, M.; Bertsch, T.; Hoffmann, U. Endothelial cell-specific molecule-1/endocan: Diagnostic and prognostic value in patients suffering from severe sepsis and septic shock. J. Crit. Care 2016, 31, 68-75. [CrossRef] [PubMed]

8. Cakmak, M.; Yilmaz, H.; Bağlar, E.; Darcin, T.; Inan, O.; Aktas, A.; Çelik, H.; Ozdemir, O.; Atalay, C.R.; Akcay, A. Serum levels of endocan correlate with the presence and severity of pre-eclampsia. Clin. Exp. Hypertens. 2015, 38, 137-142. [CrossRef] [PubMed]

9. Lee, Y.H.; Kim, J.S.; Kim, S.-Y.; Kim, Y.G.; Moon, J.-Y.; Jeong, K.-H.; Lee, T.W.; Thm, C.-G.; Lee, S.-H. Plasma endocan level and prognosis of immunoglobulin A nephropathy. Kidney Res. Clin. Pr. 2016, 35, 152-159. [CrossRef]

10. Lee, Y.H.; Kim, S.-Y.; Moon, H.; Seo, J.-W.; Kim, D.-J.; Park, S.H.; Kim, Y.-G.; Moon, J.-Y.; Kim, J.S.; Jeong, K.-H.; et al. Endocan as a marker of microvascular inflammation in kidney transplant recipients. Sci. Rep. 2019, 9, 1-12. [CrossRef]

11. Zhao, T.; Kecheng, Y.; Zhao, X.; Hu, X.; Zhu, J.; Wang, Y.; Ni, J. The higher serum endocan levels may be a risk factor for the onset of cardiovascular disease. Medicine 2018, 97, e13407. [CrossRef]

12. Qiu, C.-R.; Fu, Q.; Sui, J.; Zhang, Q.; Wei, P.; Wu, Y.; Zhu, K.; Lu, Y.; Zong, B. Serum Endothelial Cell-Specific Molecule 1 (Endocan) Levels in Patients With Acute Myocardial Infarction and Its Clinical Significance. Angiology 2016, 68, 354-359. [CrossRef] [PubMed]

13. Whayne, T.F. Endocan in hypertension and cardiovascular disease. Angiology 2013, 65, 757-759. [CrossRef] [PubMed]

14. Pawlak, K.; Mysliwiec, M.; Pawlak, D. Endocan-The new endothelial activation marker independently associated with soluble endothelial adhesion molecules in uraemic patients with cardiovascular disease. Clin. Biochem. 2015, 48, 425-430. [CrossRef] 
15. Lee, Y.H.; Shin, Y.S.; Lee, S.-Y.; Kim, Y.G.; Lee, S.-H.; Moon, J.-Y.; Jeong, K.H.; Hwang, H.S.; Ahn, S.Y.; Lee, H.J.; et al. Effects of online hemodiafiltration on anemia and nutritional status in chronic hemodialysis patients. Kidney Res. Clin. Pr. 2020, 39, 103-111. [CrossRef] [PubMed]

16. Hwang, H.S.; Kim, J.S.; Kim, Y.G.; Lee, S.-Y.; Ahn, S.Y.; Lee, H.J.; Lee, D.-Y.; Lee, S.-H.; Moon, J.-Y.; Jeong, K.H. Circulating PCSK9 Level and Risk of Cardiovascular Events and Death in Hemodialysis Patients. J. Clin. Med. 2020, 9, 244. [CrossRef]

17. Oktar, S.F.; Guney, I.; Eren, S.A.; Oktar, L.; Kosar, K.; Buyukterzi, Z.; Alkan, E.; Biyik, Z.; Erdem, S.S. Serum endocan levels, carotid intima-media thickness and microalbuminuria in patients with newly diagnosed hypertension. Clin. Exp. Hypertens. 2019, 41, 787-794. [CrossRef]

18. Balta, Ş.; Mikhailidis, D.P.; Demirkol, S.; Ozturk, C.; Ermis, N.; Demir, M.; Celik, T.; Turker, T.; Iyisoy, A. Endocan-A Novel Inflammatory Indicator in Newly Diagnosed Patients with Hypertension. Angiology 2014, 65, 773-777. [CrossRef]

19. Mihajlovic, D.M.; Lendak, D.F.; Brkic, S.V.; Draskovic, B.G.; Mitic, G.P.; Mikic, A.S.N.; Cebovic, T.N. Endocan is useful biomarker of survival and severity in sepsis. Microvasc. Res. 2014, 93, 92-97. [CrossRef]

20. Scherpereel, A.; Gentina, T.; Grigoriu, B.D.; Sénéchal, S.; Janin, A.; Tsicopoulos, A.; Plénat, F.; Béchard, D.; Tonnel, A.-B.; Lassalle, P. Overexpression of endocan induces tumor formation. Cancer Res. 2003, 63, 6084-6089.

21. Gunay, M.; Mertoglu, C. Increase of endocan, a new marker for inflammation and endothelial dysfunction, in acute kidney injury. North. Clin. Istanb. 2018, 6, 124-128. [CrossRef] [PubMed]

22. Azimi, A. Could "calprotectin" and "endocan" serve as "Troponin of Nephrologists"? Med. Hypotheses 2017, 99, 29-34. [CrossRef] [PubMed]

23. Su, Y.-H.; Shu, K.-H.; Hu, C.-P.; Cheng, C.-H.; Wu, M.-J.; Yu, T.-M.; Chuang, Y.-W.; Huang, S.-T.; Chen, C.-H. Serum Endocan Correlated With Stage of Chronic Kidney Disease and Deterioration in Renal Transplant Recipients. Transplant. Proc. 2014, 46, 323-327. [CrossRef] [PubMed]

24. Oka, S.; Obata, Y.; Sato, S.; Torigoe, K.; Sawa, M.; Abe, S.; Muta, K.; Ota, Y.; Kitamura, M.; Kawasaki, S.; et al. Serum Endocan as a Predictive Marker for Decreased Urine Volume in Peritoneal Dialysis Patients. Med. Sci. Monit. 2017, 23, 1464-1470. [CrossRef] [PubMed]

25. Poon, P.Y.-K.; Ng, J.K.-C.; Fung, W.W.-S.; Chow, K.-M.; Kwan, B.C.-H.; Li, P.K.-T.; Szeto, C.C. Relationship between Plasma Endocan Level and Clinical Outcome of Chinese Peritoneal Dialysis Patients. Kidney Blood Press. Res. 2019, 44, 1259-1270. [CrossRef] [PubMed]

26. Samouilidou, E.; Bountou, E.; Papandroulaki, F.; Papamanolis, M.; Papakostas, D.; Grapsa, E. Serum Endocan Levels are Associated with Paraoxonase 1 Concentration in Patients with Chronic Kidney Disease. Ther. Apher. Dial. 2018, 22, 325-331. [CrossRef]

27. McMillan, R.; Skiadopoulos, L.; Hoppensteadt, D.; Guler, N.; Bansal, V.; Parasuraman, R.; Fareed, J. Biomarkers of Endothelial, Renal, and Platelet Dysfunction in Stage 5 Chronic Kidney Disease Hemodialysis Patients with Heart Failure. Clin. Appl. Thromb. 2017, 24, 235-240. [CrossRef]

28. Bouillanne, O.; Morineau, G.; Dupont, C.; Coulombel, I.; Vincent, J.-P.; Nicolis, I.; Benazeth, S.; Cynober, L.; Aussel, C. Geriatric Nutritional Risk Index: A new index for evaluating at-risk elderly medical patients. Am. J. Clin. Nutr. 2005, 82, 777-783. [CrossRef]

29. Lee, W.; Ku, S.-K.; Kim, S.-W.; Bae, J.-S. Endocan Elicits Severe Vascular Inflammatory Responses In Vitro and In Vivo. J. Cell. Physiol. 2014, 229, 620-630. [CrossRef]

Publisher's Note: MDPI stays neutral with regard to jurisdictional claims in published maps and institutional affiliations.

(C) 2020 by the authors. Licensee MDPI, Basel, Switzerland. This article is an open access article distributed under the terms and conditions of the Creative Commons Attribution (CC BY) license (http://creativecommons.org/licenses/by/4.0/). 